Research Article

\title{
Medical Image Denoising Algorithm Based on Sparse Nonlocal Regularized Weighted Coding and Low Rank Constraint
}

\author{
Quan Yuan $\mathbb{D}^{1},{ }^{1}$ Zhenyun Peng $\mathbb{D}^{1},{ }^{1}$ Zhencheng Chen $\left(\mathbb{D},{ }^{1}\right.$ Yanke Guo ${ }^{(D)}{ }^{1}$ \\ Bin Yang $\mathbb{D}^{2}$, and Xiangyan Zeng $\mathbb{D}^{3}$ \\ ${ }^{1}$ School of Electronic Engineering and Automation, Guilin University of Electronic Technology, Guilin 541004, Guangxi, China \\ ${ }^{2}$ Xi'an Tapo Primary School, Chang'an District, Xi'an 710119, Shaanxi, China \\ ${ }^{3}$ School of Mathematics and Computing Science, Guilin University of Electronic Technology, Guilin 541004, Guangxi, China
}

Correspondence should be addressed to Zhencheng Chen; 1808008004@mails.guet.edu.cn

Received 6 April 2021; Revised 8 May 2021; Accepted 24 May 2021; Published 7 June 2021

Academic Editor: Gustavo Ramirez

Copyright (C) 2021 Quan Yuan et al. This is an open access article distributed under the Creative Commons Attribution License, which permits unrestricted use, distribution, and reproduction in any medium, provided the original work is properly cited.

Medical image information may be polluted by noise in the process of generation and transmission, which will seriously hinder the follow-up image processing and medical diagnosis. In medical images, there is a typical mixed noise composed of additive white Gaussian noise (AWGN) and impulse noise. In the conventional denoising methods, impulse noise is first removed, followed by the elimination of white Gaussian noise (WGN). However, it is difficult to separate the two kinds of noises completely in practical application. The existing denoising algorithm of weight coding based on sparse nonlocal regularization, which can simultaneously remove AWGN and impulse noise, is plagued by the problems of incomplete noise removal and serious loss of details. The denoising algorithm based on sparse representation and low rank constraint can preserve image details better. Thus, a medical image denoising algorithm based on sparse nonlocal regularization weighted coding and low rank constraint is proposed. The denoising effect of the proposed method and the original algorithm on computed tomography (CT) image and magnetic resonance (MR) image are compared. It is revealed that, under different $\sigma$ and $\rho$ values, the PSNR and FSIM values of CT and MRI images are evidently superior to those of traditional algorithms, suggesting that the algorithm proposed in this work has better denoising effects on medical images than traditional denoising algorithms.

\section{Introduction}

Due to the influence of imaging mechanism, external signal interference, signal attenuation in the transmission process, and other factors, medical images are doped with noise $[1,2]$. Medical imaging is affected by system requirements, patient comfort, and other practical factors, so the image acquisition should be completed in a short time. However, increased efficiency comes at a cost. The image clarity is reduced, the image details are not prominent, and the visual effect of the image is reduced, which increases the difficulty of the image analysis by doctors [3]. To remove the mixed noise of pulse noise and WGN existing in medical images, in conventional denoising methods, the impulse noise will be removed first, and then, the WGN is removed $[4,5]$. However, it is difficult to separate the two kinds of noises completely in practical application. The high-frequency information of the target in the image is often lost, resulting in blurring of edges and textures [6], so it is necessary to explore a better denoising method.

In 2014, Jielin et al. proposed a denoising method for hyperspectral images, named weighted encoding with sparse nonlocal regularization (WESNR) [7]. It can remove both AWGN and impulse noise at the same time, and the operation speed is fast. However, the noise removal is not complete and the details are lost seriously. In 2015, Zhao et al. introduced low rank constraint as regularization for image denoising and proposed a denoising algorithm based on sparse representation and low rank constraint [8]. In addition to theoretical analysis, the rationality of combining sparse representation with low rank was analyzed by the experimental method. The results showed that the proposed 
denoising method had better performance than other denoising methods. It could remove AWGN and impulse noise at the same time and could better preserve the image detail.

Thus, aiming at the situation that the denoising algorithm based on sparse nonlocal regularization-weighted coding cannot remove the noise completely and lose more details seriously, a medical image denoising algorithm based on sparse nonlocal regularization weighted coding and low rank constraint is proposed.

\section{Denoising Algorithm of Weight Coding Based on Sparse Nonlocal Regularization}

For the image $x, x_{i, j}$ is the pixel value at $(i, j)$ and $y$ is the observation image of image $x$. For the AWGN, the pixel $y_{i, j}$ in $y$ is defined as $y_{i, j}=x_{i, j}+v_{i, j}$, where $v_{i, j}$ is an independent distributed noise and follows the zero mean Gaussian distribution. For the salt and pepper impulse noise (SPIN), $\left[d_{\min }, d_{\max }\right]$ represents the dynamic range of pixel $y$ in the observation image. The probability of SPIN is $s, 0 \leq s \leq 1$; then, the probability of $y_{i, j}=d_{\min }$ is $s / 2$, and the probability of $y_{i, j}=d_{\max }$ is $s / 2$. Thus, the observation image mixed with AWGN and SPIN can be described as follows:

$$
y_{i, j}= \begin{cases}d_{\text {min }}, & P=\frac{s}{2}, \\ d_{\max }, & P=\frac{s}{2}, \\ x_{i, j}+v_{i, j}, & P=1-s .\end{cases}
$$

For image $x, x \in R^{N}, x_{i}=R_{i} x \in R^{n}$ is used to denote an image block of size $\sqrt{n} \times \sqrt{n}$, where $R_{i}$ is a matrix vector. Based on the sparse representation theory, the over-complete dictionary is used to encode the image sparsely. $x_{i}=$ $\phi \alpha_{i}$ is prescribed, and in the equation, $\alpha_{i}$ is the sparse coding vector of the nonzero matrix. The following equation can be obtained:

$$
x=\phi \alpha .
$$

In the equation, $\alpha$ is the set of all sparse coding vectors $\alpha_{i}$.

The traditional sparse representation denoising algorithm can be expressed as follows:

$$
\widehat{\alpha}=\underset{\alpha}{\arg \min }\|y-\phi \alpha\|_{2}^{2}+\lambda R(\alpha) .
$$

In the equation, $R(\alpha)$ is a regularization term corresponding to $\alpha$, and $\lambda$ is a regularization parameter.

To make the distribution of data fitting residuals more regular, the data residuals are weighted and a new loss function is adopted. Thus, a new mixed noise removal model is obtained:

$$
\widehat{\alpha}=\underset{\alpha}{\arg \min }\left\|W^{1 / 2}(y-\Phi \alpha)\right\|_{2}^{2}+\lambda R(\alpha) .
$$

In the equation, $W$ is a diagonal weight matrix with diagonal elements.
The image block $x_{i}$ and its nonlocal prediction are encoded by the given dictionary $\phi_{i}$, that is, $x_{i}=\phi_{i} \alpha_{i}$ and $\hat{x}_{i}=\phi_{i} \mu_{i}$. The coding coefficients $x_{i}$ and $\mu_{i}$ are similar. Thus, $\sum_{i}\left\|\alpha_{i}-\mu_{i}\right\|_{l}$ is regarded as the regularization term and applied to the above equation:

$$
\widehat{\alpha}=\underset{\alpha}{\arg \min }\left\|W^{1 / 2}(y-\Phi \alpha)\right\|_{2}^{2}+\lambda \sum_{i}\left\|\alpha_{i}-\mu_{i}\right\|_{l_{p}} .
$$

In the equation, $l_{p}(p=1$ or $p=2)$ is the $l_{p}$ norm.

To determine the value of $p$, it is necessary to verify the distribution of $\alpha_{i}-\mu_{i}$.

The following equation is set:

$$
\gamma_{i}=\alpha_{i}-\mu_{i}
$$

The elements in $\gamma_{i}$ are assumed to be independently identically distributed and follow the generalized Gaussian distribution, which is defined as

$$
f(\gamma)=\frac{\beta \exp \left\{-\left(|\gamma| / \sigma_{\gamma}\right)^{\beta}\right\}}{\left(2 \sigma_{\gamma} \Gamma(1 / \beta)\right)} .
$$

In the equation, $\Gamma$ is the gamma function and $\sigma_{\gamma}$ is the scalar parameter. The value of $\beta$ determines the shape of the generalized Gaussian distribution. In particular, setting $\beta=$ 1 or $\beta=2$ will lead to Laplacian or Gaussian distribution, respectively. The Laplace distribution is more consistent with the histogram of $\gamma$. Thus, $\gamma$ is approximately assumed to obey the Laplace distribution. The regularization of the norm $l_{1}$ on $\gamma$ can lead to an estimation similar to that of a map. The model is as follows:

$$
\widehat{\alpha}=\underset{\alpha}{\arg \min }\left\|W^{1 / 2}(y-\Phi \alpha)\right\|_{2}^{2}+\lambda_{1} \sum_{i}\left\|\alpha_{i}-\mu_{i}\right\|_{1} .
$$

\section{Denoising Algorithm Based on Sparse Nonlocal Regularized Weighted Coding and Low Rank Constraint}

The denoising algorithm based on sparse nonlocal regularization weight coding still has some shortcomings. When the algorithm is used to process the noise of medical images with more details, the noise removal is not complete and the details are lost seriously. Since the combination of sparse representation and low rank can remove image noise better, a low rank constraint term is added to improve the denoising performance. Thus, equation (8) can be changed into

$$
\{x, \alpha\}=\underset{x, \alpha}{\arg \min }\left\|W^{1 / 2}(y-\Phi \alpha)\right\|_{2}^{2}+\lambda_{1} \sum_{i}\left\|\alpha_{i}-\mu_{i}\right\|_{1}+\lambda_{2} \operatorname{rank}(x) .
$$

In the equation, $\lambda_{1}$ and $\lambda_{2}$ are nonnegative constants, the rank of the matrix can be regarded as the $l_{0}$ norm of its singular value matrix, which is nonconvex, and the solution of objective function (9) may not be unique. The sum of absolute values of all singular values is considered as the rank operator of kernel norm. The objective function (9) can be changed to 


$$
\{x, \alpha\}=\underset{x, \alpha}{\arg \min }\left\|W^{1 / 2}(y-\Phi \alpha)\right\|_{2}^{2}+\lambda_{1} \sum_{i}\left\|\alpha_{i}-\mu_{i}\right\|_{1}+\lambda_{2}\|x\|_{*} .
$$

In the equation, $\|\cdot\|_{*}$ is the kernel norm. In order to simulate the solution process, an auxiliary variable $U$ is introduced. The variable $x$ in the third term is replaced by a constraint $U(x=U)$. The objective function (10) can be changed into

$$
\{x, U, \alpha\}=\underset{x, \alpha}{\arg \min }\left\|W^{1 / 2}(y-\Phi \alpha)\right\|_{2}^{2}+\lambda_{1} \sum_{i}\left\|\alpha_{i}-\mu_{i}\right\|_{1}+\lambda_{2}\|U\|_{*} .
$$

For an appropriate parameter $\lambda_{3}, \lambda_{3}$ is a nonnegative constant, and the following unconstrained corresponding terms are solved by quadratic penalty function:

$$
\begin{aligned}
\{x, U, \alpha\}= & \underset{x, U, \alpha}{\arg \min }\left\|W^{1 / 2}(y-\Phi \alpha)\right\|_{2}^{2}+\lambda_{1} \sum_{i}\left\|\alpha_{i}-\mu_{i}\right\|_{1} \\
& +\lambda_{2}\|U\|_{*}+\lambda_{3}\|x-U\|_{2}^{2} .
\end{aligned}
$$

For the objective function (12), an alternative optimization method is used. Some variables in (12) are optimized, and then, they are reduced to the following problems.

$\alpha$ is optimized by fixing $U$ and $x$ :

$$
\{\alpha\}=\underset{\alpha}{\arg \min }\left\|W^{1 / 2}(y-\Phi \alpha)\right\|_{2}^{2}+\lambda_{1} \sum_{i}\left\|\alpha_{i}-\mu_{i}\right\|_{1} .
$$

$U$ is optimized by fixing $\alpha$ and $x$ :

$$
\{U\}=\underset{U}{\arg \min } \frac{\lambda_{2}}{2 \lambda_{3}}\|U\|_{*}+\frac{1}{2}\|x-U\|_{2}^{2} .
$$

$x$ is optimized by fixing $\alpha$ and $U$ :

$$
\{x\}=\underset{x}{\arg \min }\left\|W^{1 / 2}(y-\Phi \alpha)\right\|_{2}^{2}+\lambda_{3}\|x-U\|_{2}^{2} .
$$

Dictionary selection is an important issue in signal sparse coding and reconstruction [9]. In particular, learning dictionaries from natural image patches has shown encouraging results in image restoration. In the groundbreaking work of K-Singular Value Decomposition (SVD), a complete general dictionary is learned to handle any input patch [10]. However, $\mathrm{K}-\mathrm{SVD}$ is not suitable for the content of a given patch, and its efficiency is not high in practical application. A set of local principal component analysis (PCA) dictionaries learned from natural images is adopted [11].

Once $W$ is given, the sparse nonlocal regularized weighted coding model in the equation can be obtained. The problem becomes the $l_{1}$ norm sparse coding problem, which is solved by iterative weighting scheme. $V$ is set to be a diagonal matrix. After $k+1$ iterations, each element of $V$ is updated as follows:

$$
V_{i i}^{(k+1)}=\frac{\lambda}{\left(\left(\alpha_{i}^{(k)}-\mu_{i}\right)^{2}+\varepsilon^{2}\right)^{1 / 2}} .
$$

In the equation, $\varepsilon$ is a scalar and $\alpha_{i}^{k}$ is the $i$ th element of the coding vector $\alpha$ in the $k$ th iteration. The sparse code $\alpha$ is updated by the following function:

$$
\alpha^{k}=\left(\Phi^{T} W \Phi+V^{(k)}\right)^{-1}\left(\Phi^{T} W y-\Phi^{T} W \Phi \mu\right)+\mu .
$$

According to equation (14), the low rank constraint term is optimized:

$$
\left\{U^{k}\right\}=\underset{U}{\arg \min } \frac{\lambda_{2}}{2 \lambda_{3}}\|U\|_{*}+\frac{1}{2}\left\|x^{k-1}-U\right\|_{2}^{2} .
$$

In the equation, $x^{k-1}=W^{*} \sum V$ (singular value decomposition). The iterative equation is as follows:

$$
U^{k}==W^{*} S_{\tau}(\Sigma) V .
$$

In the equation, $S_{\tau}(\cdot)$ is a soft contraction operator and $S_{\tau}(\sigma)=\operatorname{sign}(\sigma) \cdot \max (\sigma-\tau, 0) \quad$ is a soft threshold algorithm.

According to equation (15), the image restoration can be optimized. There is a closed solution of $x$ in the above equation, that is,

$$
x=\left(I+\lambda_{3} I\right)^{-1} \cdot\left(\Phi \alpha+\lambda_{3} U\right) .
$$

The iterative equation is as follows:

$$
x^{k}=\left(I+\lambda_{3} I\right)^{-1} \cdot\left(\Phi \alpha^{k}+\lambda_{3} U^{k}\right) .
$$

The above optimization for the problem is repeated until the iteration stop condition is satisfied. When there is no significant change in the solution of continuous iteration or the corresponding objective function value, that is, the difference norm between the two successive iterative solutions is less than the given positive norm, the algorithm stops and remains unchanged. If the running time exceeds the upper limit, the iteration process can also be terminated. In the experiment, when the number of iterations reaches a predetermined maximum, the iteration is stopped. The proposed denoising algorithms are summarized in Algorithm 1.

\section{Experiment}

To enhance the persuasiveness of this algorithm, in addition to the comparison of visual effects, the following criteria are set for numerical values: peak signal-to-noise ratio (PSNR) and feature similarity index (FSIM), and the higher the value of PSNR and FSIM is, the higher the reconstruction quality is [12].

A brain CT image of $512 \times 512$ pixels is selected as the experimental object. For the mixed noise with different proportions of WGN and SPIN, the reconstruction effect and numerical comparison of various algorithms under nine kinds of noise situations are listed, as shown in Figure 1 and Table 1. The black and bold font in Table 1 indicates the advantages of the algorithm.

For CT images with nine different proportions of WGN and SPIN, the reconstruction effect and index values of the proposed algorithm are better than WESNR algorithm, and the proposed algorithm has better performance in detail 
Input: noisy image $y$, and the maximum iterative value $K_{\max }$

Output: denoised image data

Initialization: $x^{0}=y$, initializing model parameters, $k=1$

while $k \leq K_{\max }$ do

(1) Calculation:

$\alpha^{k}=\left(\Phi^{T} W \Phi+V^{(k)}\right)^{-1}\left(\Phi^{T} W y-\Phi^{T} W \Phi \mu\right)+\mu$

(2) Calculation:

$$
U^{k}==W^{*} S_{\tau}(\Sigma) V
$$

(3) Calculation:

$$
x^{k}=\left(I+\lambda_{3} I\right)^{-1} \cdot\left(\Phi \alpha^{k}+\lambda_{3} U^{k}\right)
$$

(4) $k=k+1$

end while

Algorithm 1: Denoising algorithm based on sparse nonlocal regularized weighted coding and low rank constrain (flowchart of denoising algorithm).

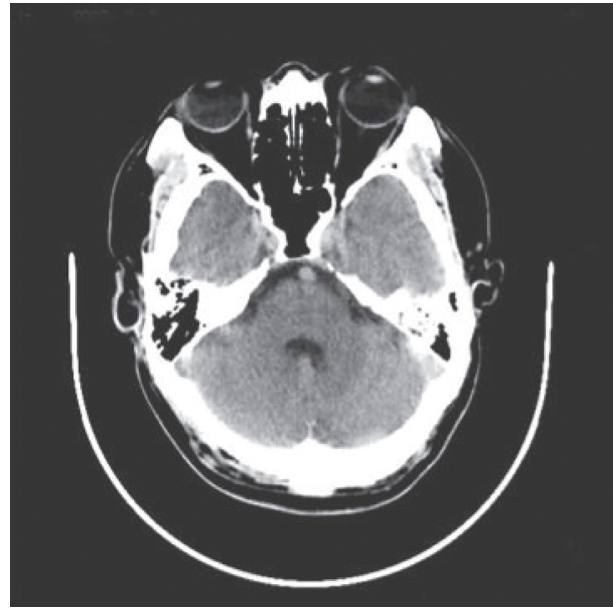

(a)

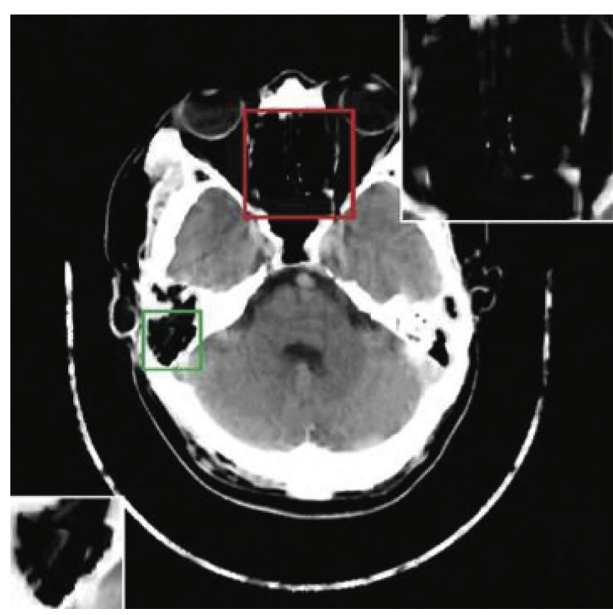

(c)

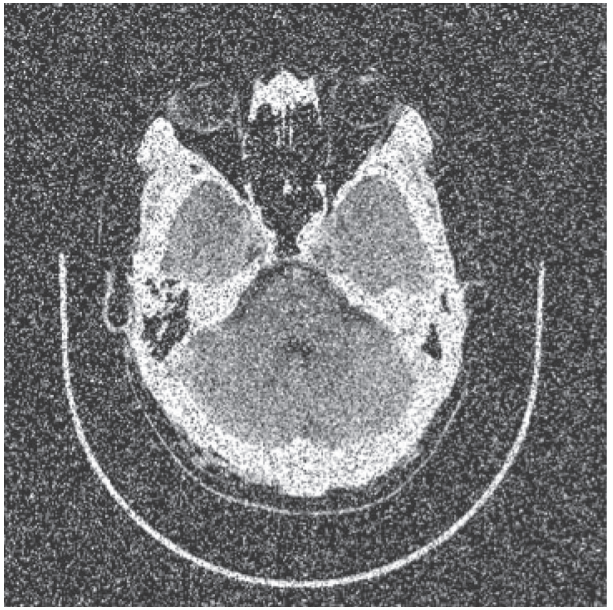

(b)

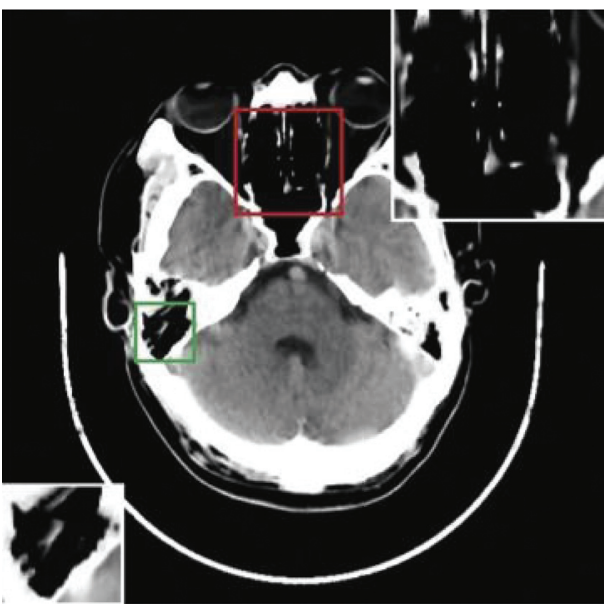

(d)

FIGURE 1: Comparison of CT images based on different algorithms. (a) CT image without noise added; (b) image with noise added when $\sigma=10$ and $\rho=30 \%$; (c) the effect of WESNR algorithm; (d) the effect of the proposed algorithm; the red and green boxes indicate that the area has been enlarged. 
TABLE 1: Comparison of PSNR and FSIM after CT image denoising.

\begin{tabular}{|c|c|c|c|c|c|}
\hline \multirow{2}{*}{$\sigma$} & \multirow{2}{*}{$\rho(\%)$} & \multicolumn{2}{|c|}{ WESNR } & \multicolumn{2}{|c|}{ Proposed algorithm } \\
\hline & & PSNR & FSIM & PSNR & FSIM \\
\hline \multirow{3}{*}{10} & 30 & 22.53 & 0.9442 & 22.66 & 0.9459 \\
\hline & 40 & 20.27 & 0.9220 & 20.80 & 0.9277 \\
\hline & 50 & 18.38 & 0.8914 & 18.65 & 0.8958 \\
\hline \multirow{3}{*}{20} & 30 & 21.09 & 0.9196 & 21.61 & 0.9233 \\
\hline & 40 & 19.19 & 0.8944 & 19.45 & 0.8958 \\
\hline & 50 & 17.41 & 0.8627 & 17.93 & 0.8698 \\
\hline \multirow{3}{*}{30} & 30 & 18.22 & 0.8679 & 19.71 & 0.8913 \\
\hline & 40 & 17.63 & 0.8544 & 17.76 & 0.8586 \\
\hline & 50 & 16.94 & 0.8368 & 17.06 & 0.8399 \\
\hline
\end{tabular}

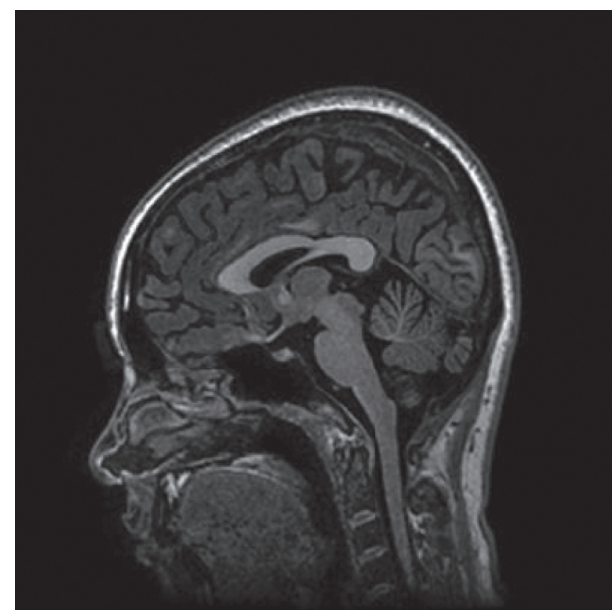

(a)

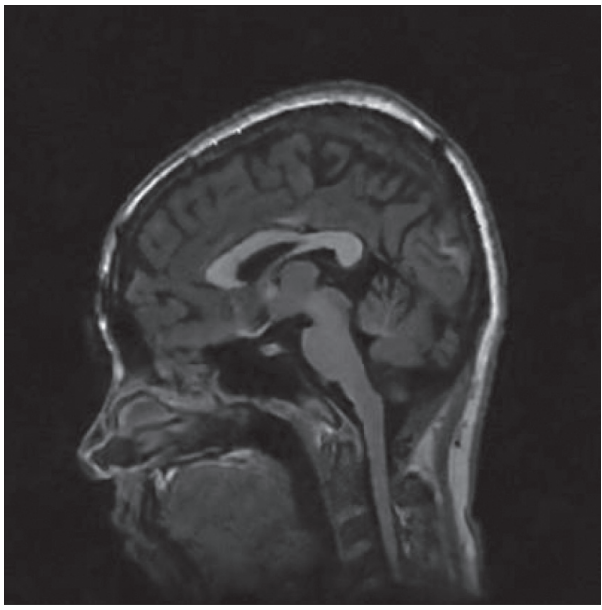

(c)

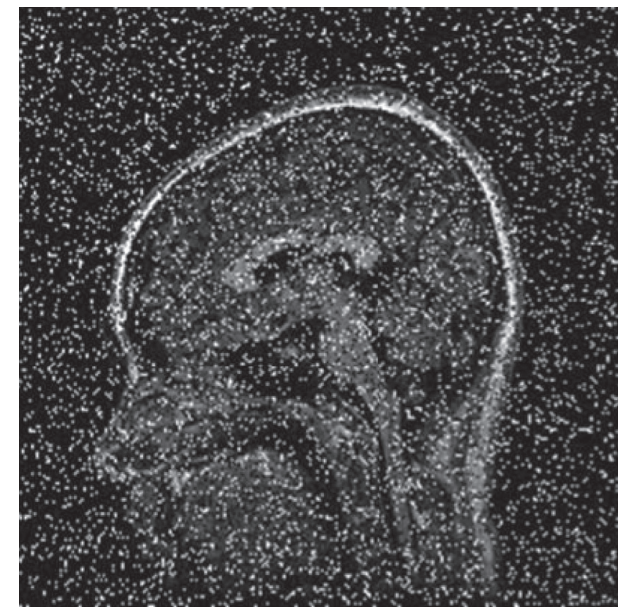

(b)

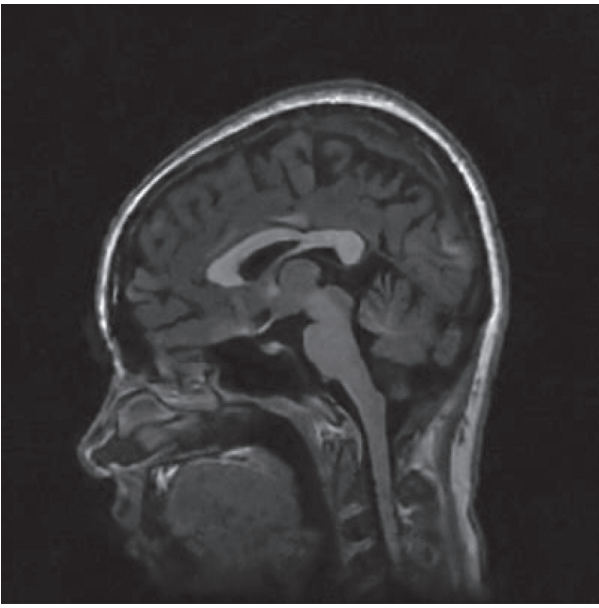

(d)

FIgURE 2: Comparison of MRI images based on different algorithms. (a) MR image without noise added; (b) image with noise added when $\sigma=10$ and $\rho=30 \%$; (c) the effect of WESNR algorithm. 3D figure is the effect of the proposed algorithm.

retention and noise removal, which is consistent with the study results of $\mathrm{Wu}$ et al. [13].

A brain MR image of $256 \times 256$ pixels is selected as the experimental object. For the mixed noise with different proportions of WGN and SPIN, the reconstruction effect and numerical comparison of various algorithms under nine kinds of noise situations are listed, as shown in Figure 2 and Table 2. The black and bold font in Table 2 shows the advantages of the algorithm.

For MR images with nine different proportions of WGN and SPIN, the reconstruction effect and index values of the proposed algorithm are better than WESNR algorithm, and 
TABle 2: Comparison of PSNR and FSIM after MR image denoising.

\begin{tabular}{cccccc}
\hline & & \multicolumn{2}{c}{ WESNR } & \multicolumn{2}{c}{$\begin{array}{c}\text { Proposed } \\
\text { algorithm }\end{array}$} \\
& $\rho(\%)$ & PSNR & FSIM & PSNR & FSIM \\
\hline \multirow{3}{*}{10} & 30 & 28.42 & 0.8901 & $\mathbf{2 9 . 6 3}$ & $\mathbf{0 . 8 9 5 1}$ \\
& 40 & 27.50 & 0.8793 & $\mathbf{2 8 . 4 4}$ & $\mathbf{0 . 8 8 3 2}$ \\
& 50 & 25.19 & 0.8561 & $\mathbf{2 5 . 4 3}$ & $\mathbf{0 . 8 6 1 8}$ \\
\hline \multirow{3}{*}{20} & 30 & 26.68 & 0.8334 & $\mathbf{2 6 . 8 1}$ & $\mathbf{0 . 8 3 3 9}$ \\
& 40 & 25.08 & 0.8192 & $\mathbf{2 5 . 6 5}$ & $\mathbf{0 . 8 1 9 6}$ \\
& 50 & 23.72 & 0.8005 & $\mathbf{2 3 . 8 6}$ & $\mathbf{0 . 8 1 0 1}$ \\
\hline \multirow{3}{*}{30} & 30 & 24.83 & 0.8051 & $\mathbf{2 5 . 2 4}$ & $\mathbf{0 . 8 0 5 9}$ \\
& 40 & 22.66 & 0.7878 & $\mathbf{2 3 . 2 7}$ & $\mathbf{0 . 7 8 9 1}$ \\
& 50 & 21.55 & 0.7654 & $\mathbf{2 1 . 7 3}$ & $\mathbf{0 . 7 6 8 1}$ \\
\hline
\end{tabular}

the proposed algorithm has better performance in detail retention and noise removal, which is consistent with the study results of Cai et al. [14].

\section{Conclusion}

A medical image denoising algorithm based on sparse nonlocal regularized weighted coding and low rank constraint is proposed, which can remove AWGN and impulse noise at one time and ensure the retention of details and noise processing effect. Experiments on CT and MR images with noise show that the proposed algorithm is superior to the original algorithm in terms of visual effect and PSNR and FSIM values. Although the effect of the proposed algorithm is rather favorable, the running time is relatively long, and it is only compared with the WESNR algorithm. Therefore, the algorithm needs to be further optimized to improve time efficiency in the subsequent research, and performance comparison and analysis with more denoising algorithms should be performed.

\section{Data Availability}

No data were used to support this study.

\section{Conflicts of Interest}

The authors declare that they have no conflicts of interest.

\section{Acknowledgments}

This work was supported by research supported by National Natural Science Foundation of China (11961012 and 71801060), Provincial Natural Science Foundation of Guangxi (2018GXNSFBA281192，2017GXNSFAA198248, and 2017GXNSFBA198182), and National Major Scientific Research Instruments Development Program (61627807).

\section{References}

[1] P. Gravel, G. Beaudoin, and J. A. DeGuise, "A method for modeling noise in medical images," IEEE Transactions on Medical Imaging, vol. 23, no. 10, pp. 1221-1232, 2004.
[2] B. Shinde, D. Mhaske, M. Patare et al., "Noise detection and noise removal techniques in medical images," Indian Journal of Computer Ence \& Engineering, vol. 3, no. 1, pp. 51-60, 2012.

[3] G. Dougherty, Digital Image Processing for Medical applications, Cambridge University Press, Cambridge, UK, 2009.

[4] G. Ma, V. Vidal, G. Verdú et al., "Medical image restoration with different types of noise," in Proceedings of the Annual International Conference of the IEEE Engineering in Medicine \& Biology Society, San Diego, CA, USA, August 2012.

[5] B. Christian, D. R. Haynor, and P. Hellier, Proceedings of Medical Image Computing and Computer-Assisted Intervention-MICCAI 2004, Springer Berlin Heidelberg, Berlin, Germany, 2010.

[6] L. Xuegang, L. Junrui, and W. Juan, "Nonconvex low rank approximation with phase congruency regularization for mixed noise removal," IEEE Access, vol. 7, pp. 179538-179551, 2019.

[7] J. Jielin, L. Jian Yang, and J. Yang, "Mixed noise removal by weighted encoding with sparse nonlocal regularization," IEEE Transactions on Image Processing, vol. 23, no. 6, pp. 26512662, 2014.

[8] Y. Q. Zhao and J. Yang, "Hyperspectral image denoising via sparse representation and low-rank constraint," IEEE Transactions on Geoence \& Remote Sensing, vol. 53, no. 1, pp. 296-308, 2014.

[9] G. J. Power, M. A. Karim, and F. Ahmed, "Charting image artifacts in digital image sequences using velocital information content," Proceedings of SPIE, vol. 3460, pp. 642-651, 1998.

[10] L. S. Davis, "Label consistent K-SVD: learning a discriminative dictionary for recognition," IEEE Computer Society, vol. 35, 2013.

[11] R. Rubinstein, M. Zibulevsky, and M. Elad, "Double sparsity: learning sparse dictionaries for sparse signal approximation," IEEE Transactions on Signal Processing, vol. 58, no. 3, pp. 1553-1564, 2010.

[12] U. Sara, M. Akter, and M. S. Uddin, "Image quality assessment through FSIM, SSIM, MSE and PSNR-a comparative study," Journal of Computer and Communications, vol. 7, no. 3, pp. 8-18, 2019.

[13] G. Wu, Z. Shi, Y. Chen et al., "A sparse representation-based radiomics for outcome prediction of higher grade gliomas," Medical Physics, vol. 46, no. 1, pp. 250-261, 2019 Jan.

[14] S. Cai, K. Liu, M. Yang, J. Tang, X. Xiong, and M. Xiao, “A new development of non-local image denoising using fixed-point iteration for non-convex ep sparse optimization," PLoS One, vol. 13, no. 12, Article ID e0208503, 2018. 